\title{
TORTICOLIS MUSCULAR CONGÉNITO DE CAUSA POSTURAL E INTRAUTERINO: REPORTE DE UN CASO
}

\author{
Custodio Olsen Quispe Condori ${ }^{1,2}$ Carmen Del Carmen Ramos ${ }^{3}$.
}

\begin{abstract}
RESUMEN
El tortícolis muscular congénito es una entidad clínica conocida desde la antigüedad, se caracteriza por presentar una desviación de la cabeza con inclinación y rotación hacia el lado afecto, el mentón gira en sentido contrario; que produce a lo largo de la vida un defecto funcional importante del cuello y un defecto estético considerable. Los factores de riesgo son: obstétricos, maternos y fetales. Se presenta un caso clínico de una recién nacida de parto por cesárea con tortícolis muscular congénito de causa postural e intrauterino, que se evidencia con las vistas fotográficas; cuyo pronóstico es bueno cuando se inicia pronto el manejo fisioterapéutico. De las revisiones hechas, no existen datos sobre el diagnóstico y manejo del tortícolis muscular congénito. El presente trabajo realizado en el Instituto Nacional Materno Perinatal, tiene por objetivo contribuir al conocimiento médico e intercambiar experiencias en el diagnóstico precoz del recién nacido con tortícolis muscular congénito de causa postural e intrauterino y la referencia oportuna un centro especializado para su manejo correspondiente.
\end{abstract}

Palabras clave: Tortícolis muscular congénita; Músculo del esternocleidomastoideo; Distonía Cervical (Fuente: DeCS BIREME).

\section{CONGENITAL MUSCULAR TORTICOLLIS POSTURAL AND INTRAUTERINE CAUSE: CASE REPORT}

\begin{abstract}
Congenital muscular torticollis is a well-known clinical entity since antiquity. It is characterized by a deviation of the head with tilt from the affected side; the chin rotates in the opposite direction, which occurs throughout life an important functional defect neck and considerable aesthetic defect. The risk obstetrical factors included obstetrical, maternal and fetal. A case of a newborn cesarean delivery with congenital muscular torticollis of cause postural and intrauterine, evidenced by the photo views, whose prognosis is good when the physiotherapy management starts as soon arises. The present work made in the Instituto Nacional Materno Perinatal he has objective to contribute to medical knowledge and exchanging experiences in the early diagnosis of a newborn with congenital muscular torticollis of cause postural and intrauterine and timely referral to a specialized center for its management
\end{abstract}

Key words: Congenital muscular torticollis; Sternocleidomastoid muscle; Cervical dystonia (Source: MeSH NLM).

\section{INTRODUCCIÓN}

El cuerpo humano posee una simetría bilateral característica (simetría axial o vertical), que consiste en que una parte del cuerpo se corresponde con la contralateral, es decir, que la parte izquierda del cuerpo es semejante a la derecha ${ }^{1}$. El tortícolis muscular congénito (TMC) es una entidad clínica conocida desde la antigüedad, consistente en un acortamiento del músculo esternocleidomastoideo (ECM) asociado o no a tumoración del vientre muscular, que produce a lo largo de la vida un defecto funcional importante del cuello y un defecto estético considerable y es la tercera anomalía músculo-esquelética más frecuente, después de la luxación congénita de cadera y del pie zambo. La mayoría de los profesionales atribuyen como causa del TMC a los partos traumáticos, su incidencia varía de 0,3 al $1,9 \%$ y es más frecuente en varones ${ }^{1,2}$.

Factores de riesgo: obstétricos como los partos dificultosos; maternos la primiparidad, fibromiomas, oligoamnios; fetales la macrosomía fetal ${ }^{1,3}$. Causas: el
TMC de causa postural e intrauterino, es la más frecuente y es consecuencia del espacio físico intrauterino reducido del que dispone el producto asociado a la posición que adopta el feto por largo tiempo en el útero de la mujer embarazada, lo que provocaría un acortamiento del músculo ECM, y que no está asociado a una tumoración a lo largo del músculo ECM afecto, que se manifiesta clínicamente al nacer. En cambio el TMC de causa traumática es la menos frecuente y está asociado a una tumoración en el músculo del ECM producido por un traumatismo durante el parto, por el excesivo estiramiento del músculo ECM que produce un desgarro o rotura de fibras, con formación de hematoma en forma y tamaño de una aceituna, con posterior cicatrización y acortamiento del músculo ECM que se manifiesta clínicamente alrededor de la segunda o tercera semana de vida ${ }^{2,3,4}$. Manifestación clínica el cuadro clínico se caracteriza por una inclinación con rotación de la cabeza hacia el lado afecto con acortamiento del músculo ECM asociado o no a la presencia de una tumoración en el vientre muscular, con asimetría de frente, cara y cuello;

Servicio de Puericultura, Departamento de Neonatología, del Instituto Nacional Materno Perinatal.

Departamento Académico de Pediatría de la Facultad de Medicina de la Universidad Nacional Mayor San Marcos. Lima-Perú.

Enfermera del Centro Quirúrgico del Instituto Nacional Materno Perinatal

Recibido: 14-01-15 Aprobado: 12-08-15 
en cuanto a la asimetría de la frente puede presentar leve aplanamiento oblicuo del lado afecto; en la asimetría de cara presenta poco desarrollo de la región orbitaria, malar y del ángulo mandibular del lado afecto y en la región nasal se observa desviación del tabique nasal hacia el lado afecto con asimetría del vestíbulo de las fosas nasales; en cuanto a la asimetría del cuello se observa en el lado afecto: inclinación de la cabeza sobre el cuello, con hundimiento de la zona del músculo ECM, fosa clavicular con leve depresión, disminución de la distancia cuello-hombro, hombro ligeramente elevado. El mentón gira hacia el lado no afecto. Disminución de la movilidad activa y pasiva como la flexión, extensión y rotación de la columna cervical. Asimismo, si el cuadro clínico es de consideración, pueden producirse defectos en el crecimiento de las vértebras cervicales debido a la mal posición mantenida durante largo tiempo. No existe relación entre la severidad del cuadro clínico con el modo de nacimiento, por observarse tanto en el recién nacido (RN), de parto vaginal como de parto por cesárea. La posición lateralizada de la cabeza es lo que motiva la consulta médica ${ }^{1,5,6}$. Figura 1. Diagnóstico debe incluir una exploración clínica, neurológica con el estudio ecográfico; además, de realizar el diagnóstico diferencial correspondiente. La ecografía es el procedimiento de elección para el estudio de los tortícolis. En los casos de causa postural e intrauterino, la detección de una contractura o constricción del músculo $\mathrm{ECM}$ del lado afecto y en los casos de tortícolis de causa traumática o adquirida la detección de una tumoración o masa fibrosa en el músculo ECM afecto. La ecografía es un indicador paraclínico para apreciar la severidad y el pronóstico del tortícolis ${ }^{6}$ algunas veces se solicita la radiografía cervical en busca de anomalías óseas y en casos seleccionados la tomografía axial computarizada y resonancia magnética nuclear 1,7,8. Manejo: a cargo de los profesionales especializados en rehabilitación y fisioterapia para los ejercicios de estiramiento y movimiento del cuello como la flexión, extensión y rotación; así como, el tratamiento postural y educación a los padres para que vayan realizando el trabajo en casa 1,8,9. Pronóstico es bueno, cuando el diagnóstico y el tratamiento son inmediatos, en casos severos puede necesitar un tratamiento quirúrgico ${ }^{8,9}$. El presente trabajo realizado en el Instituto Nacional Materno Perinatal tiene por Objetivo contribuir al conocimiento médico e intercambiar experiencias en el diagnóstico precoz del recién nacido con tortícolis muscular congénito de causa postural e intrauterino y la referencia oportuna a un centro especializado para su manejo correspondiente. La madre de la RN dio su consentimiento para la presente publicación.

\section{CASO CLÍNICO}

RN de parto por cesárea, el día 13 de junio 2 014, de sexo femenino, peso $3834 \mathrm{~g}$, talla $51 \mathrm{~cm}$, perímetro cefálico $35 \mathrm{~cm}$, perímetro torácico de $36 \mathrm{~cm}$, Apgar normal. Líquido amniótico claro y escaso. Datos de la madre: edad 22 años, G2P0010, un aborto hace tres años, control prenatal dos veces, Peso preconcepcional $70 \mathrm{~kg}$, peso momentos antes del parto $93 \mathrm{~kg}$, talla 155 $\mathrm{cm}$. Análisis laboratoriales, Presión arterial y Temperatura dentro de los valores referenciales. Ecografía obstétrica de 27 de mayo 2 014: 37 semanas, ponderado fetal 3 293 gramos, líquido amniótico conservado. Diagnóstico preoperatorio: gestante 2 nulípara de 39 semanas por última regla, feto valioso, ruptura de membranas una hora, oligoamnios severo e insuficiencia placentaria

Examen físico al nacer: buen estado general y al examen preferencial se observa inclinación de la cabeza hacia el lado izquierdo, impresiona cuello corto, se observan asimetría de cara y de cuello. En cuanto a la asimetría facial: se encuentra la región orbitaria y malar del lado izquierdo afectado en su desarrollo; asimismo, se observa el compromiso del desarrollo del ángulo mandibular izquierdo, comparado con el lado derecho. En la región nasal se observa desviación del tabique nasal hacia lado izquierdo y vestíbulo de la fosa nasal izquierda mayor que el derecho. En cuanto a la asimetría del cuello: se observa inclinación con ligera rotación de la cabeza sobre el lado izquierdo con hundimiento de la zona del músculo ECM izquierdo, disminución de la distancia cuello-

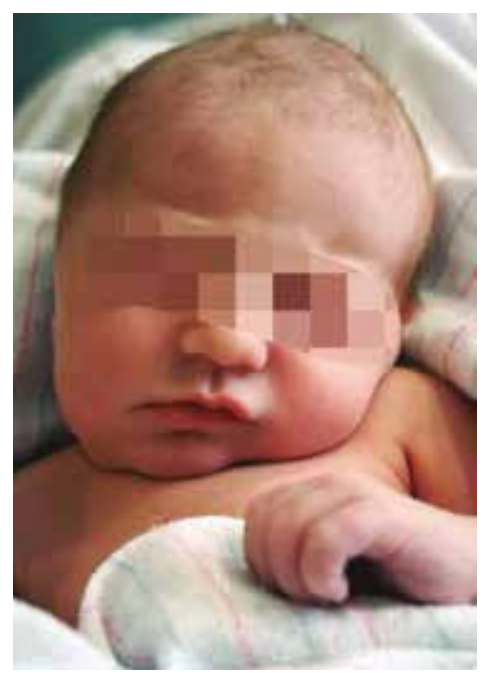

Figura 1. RN con tortícolis muscular congénito izquierdo.

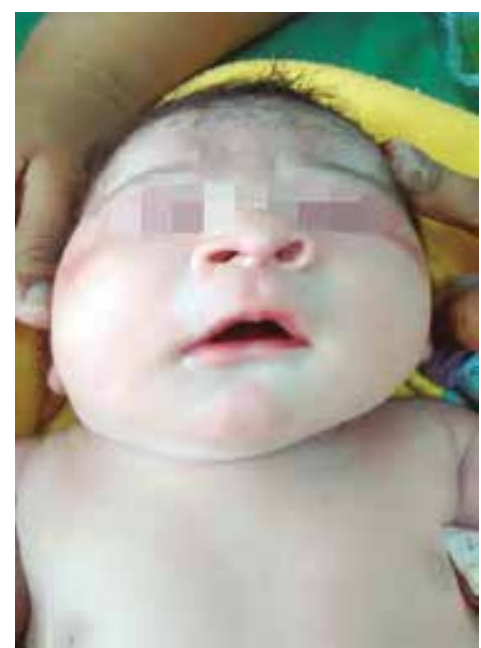

Figura 2. RN con tortícolis muscular congénita izquierdo. Vista de frente. 


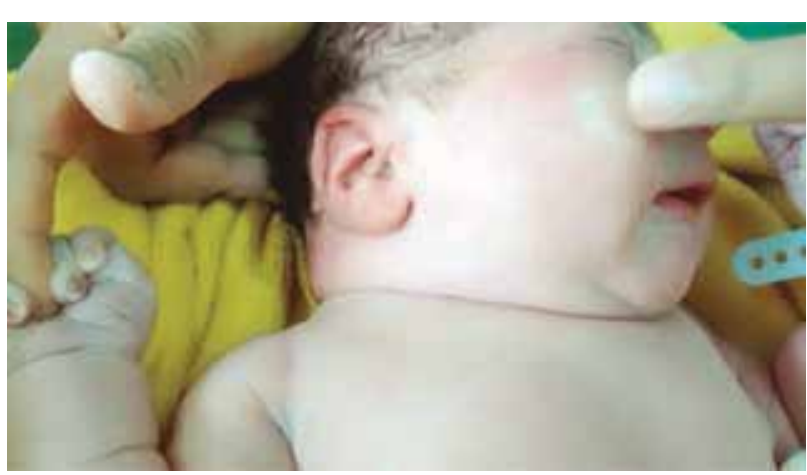

Figura 3. RN con tortícolis muscular congénito Izquierdo. Vista de cuello, lado no afecto.

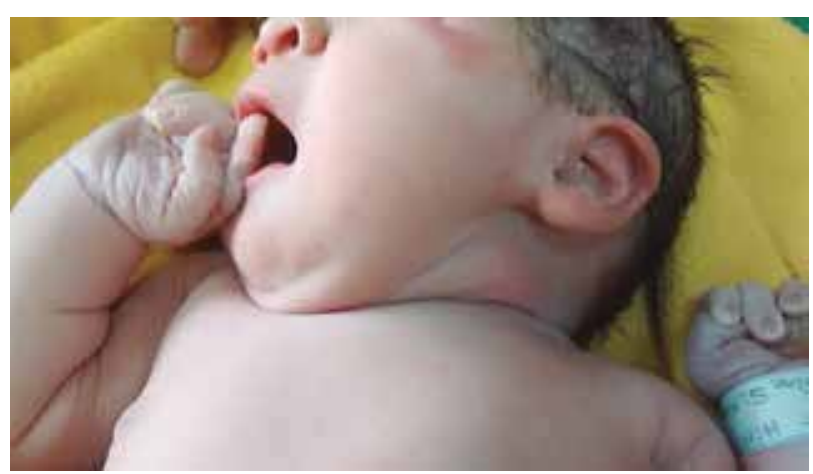

Figura 4. RN con tortícolis muscular congénito Izquierdo. Vista de cuello, lado afecto.

hombro del lado izquierdo, mentón lateralizado hacia el lado derecho, fosa clavicular izquierda con leve depresión, hombro izquierdo ligeramente elevado y disminución de la movilidad activa y pasiva de la columna cervical. Figuras $2,3,4$.

Diagnóstico al nacer: RN de término de 39 semanas. Grande para la Edad Gestacional. RN de cesárea. Deformación congénita: tortícolis muscular congénito de lado izquierdo.

\section{DISCUSIÓN}

EI TMC de causa postural e intrauterino es una alteración músculo esquelético benigno que con frecuencia, se asocia a otras anomalías ortopédicas ${ }^{1,2,7,10}$. El sitio anatómico de la enfermedad regularmente está confinado al músculo ECM, figura $5^{1,8,10}$. En el RN, como en el presente caso, lo que llama más la atención en el examen físico a la inspección es la inclinación anormal con rotación de la cabeza sobre el lado izquierdo del cuello, con hundimiento de la zona del ECM izquierdo ${ }^{1,5,6}$. Esta deformación congénita es poco valorada por el profesional que atiende al RN, para referir a un centro especializado como el Instituto Nacional de Rehabilitación en cuyas guías del año 2012, reportan la atención de ocho casos en el año $2011^{11}$, lo que refleja que estos casos no se están derivando oportunamente como

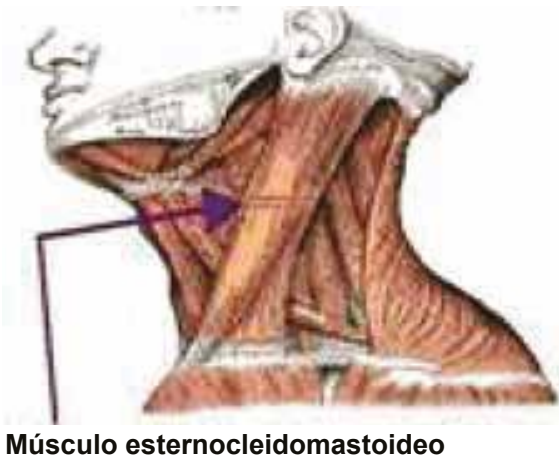

Figura 5. Músculo esternocleidomastoideo izquierdo

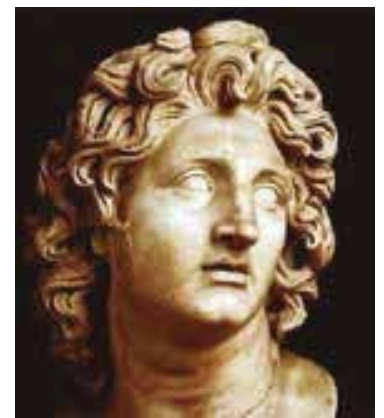

Figura 6. Secuela de tortícolis muscular congénita izquierda

debiera ser. Teniendo en cuenta que en el año 2013, en el Instituto Nacional Materno Perinatal hubo 17089 nacidos vivos ${ }^{12}$ y en el Departamento de Lima en el año 2012, hubo 195756 nacimientos ${ }^{13}$.

Presentamos el caso por tratarse una RN con TMC de causa postural e intrauterino, de parto por cesárea, grande para su edad gestacional, con un peso de 3834 $\mathrm{g}$, madre nulípara con oligoamnios severo. Conviene comentar lo siguiente: si la niña al nacer hubiera tenido un peso promedio de $3250 \mathrm{~g}$ el cuadro clínico de tortícolis no sería evidente o de estar presente sería leve o imperceptible; pero, al ser una niña grande para la edad gestacional, asociado a oligoamnios severo, la niña presentó el cuadro clínico antes descrito. Por otro lado, si la niña al nacer hubiera tenido un peso mayor, el cuadro clínico también sería mayor, pudiendo llegar a presentar un caso severo de tortícolis, el cual hubiera requerido un manejo quirúrgico ${ }^{1,7,9,14}$. La ecografía es el procedimiento de elección para el diagnóstico de TMC ${ }^{10,14,15}$, pero en otros casos similares de TMC de causa postural e intrauterino se solicitó ecografías con reportes diferentes a lo que se espera, como la presencia de ganglios o quistes y no la falta de desarrollo del músculo ECM debido a la constricción intrauterina $6,8,16$.

En cuanto al TMC de causa traumático es poco frecuente y las evidencias sobre esta causa son escasas 1,4,7,16-18.

Sin embargo, frente a un caso de TMC en el RN, la mayoría de los profesionales lo asocian con una causa traumática, hecho que contribuye, que en los informes de 
las ecografías solicitadas se encuentren incongruencias, tanto es así, que frente a un hematoma palpable del músculo ECM reportan la existencia de quistes, ganglios o adenitis causando confusión y preocupación a los pacientes.

Se concluye que el caso presentado se trata de una RN con TMC de causa postural e intrauterino debido a la posición anómala de la cabeza fetal por la restricción del espacio físico intrauterino para su desarrollo normal $\left.\right|^{4,6,16,18}$ y es pertinente recordar a los profesionales que atienden a los neonatos realizar el diagnóstico precoz de TMC e informar a la familia el defecto funcional importante del cuello y el defecto estético considerable, para la referencia oportuna a un centro de atención especializado como el Instituto Nacional de Rehabilitación, a fin de disminuir secuelas como el que se aprecia en la figura 6 .

\section{REFERENCIAS BIBLIOGRÁFICAS}

1. Lacruz-Rengel MA, Romero A, Márquez-Albornoz C, Rojas de Hernández M. Características clínicas y evolutivas del tortícolis muscular congénito. Arch Venez Puer Ped. Caracas mar.2013; 76 (1):12-16.

2. Vial I, García C, Accorsi E, Zúñoga S. Tortícolis muscular congénita. Hallazgos ultrasonográficos. Rev. Chil. Pediatr. 1995; 66(3): 156-161.

3. Cheng JCY, Metreweli C, Chen TMK, Tang S P. Correlation of ultrasonographic imaging of congenital muscular torticollis with clinical assessment in infants. Ultrasound in Med \& Biol., 2000; vol 26(8): 1237-1241.

4. Cheng JCY, Wong MWN, Tang MBBS, Chen TMK, Shum SLF, Wong EMC. Clinical Determinants of the Outcome of Manual Strertching in the Treatment of Congenital Muscular Torticollis in Infants. The Journal of Bone \& Joint Surgery. May 2001; vol 83 (5): 679-687.

5. Dudkiewics I, Ganel A, Blankstein A. Congenital Muscular torticollis In Infants: Ultrasound-Assisted Diagnosis and Evaluation. J Pediatr Orthop November/December 2005; vol 25(6):812-814.
6. Lee Y, Cho SK, Yoon K, Shin HK, Kim E, Kim Y et al, Risk factors for intrauterine constraint are associated with ultrasonographic detected severe fibrosis in early congenital muscular torticollis. Journal of Pediatric Surgery 2011; 46: 514-519.

7. Cerda J, García C. Caso clínico-radiológico. Rev Chil Pediatr 2005; 76(5): 518-520.

8. Tomczak KK, Rosman NP. Torticollis. Journal Child Neurol 2013; 28: 365-378.

9. Lee JK, Moon HJ, Park MS, Yoo WJ, Choi IH, Cho T. Change of Craniofacial Deformity After Sternocleidomastoid Muscle Release in Pediatric Patients with Congenital Muscular Torticollis. J Bone Joint Surg Am 2012; 94:e93 (1-7).

10. Haque S, Bilal BB, Kaleem M. Imaging of Torticollis in Children. RadioGraphics 2012; 32:557-571.

11. Perú. Ministerio de Salud. Instituto Nacional De Rehabilitación. Guía de Práctica Clínica Manejo de Rehabilitación en Tortícolis Muscular Congénita. Perú; MINSA; 2012.

12. Perú. Ministerio de Salud. Instituto Nacional Materno Perinatal. Oficina de Estadística e Informática .Boletín Estadístico. 2013; p 30.

13. Perú. Instituto Nacional De Estadística E Informática (INEI). Nacimientos, Defunciones, Matrimonios y Divorcios 2012. Lima, noviembre 2013; p. 19.

14. Chen M, Chang H, Hsieh C, Yen M. Predictive Model for Congenital Muscular Torticollis: Analysis of 1021 Infants With Sonography. Arch Phys Med Rehabil 2005; 86: 2199-203.

15. Park HJ, Kim SS, Lee SY, Lee YT, Yoon K, Chung EC et al. Assessment of Follow-Up Sonography and Clinical Improvement among infants with Congenital Muscular Torticollis. Am J Neuroradiol Apr 2013; 34: 890-894

16. Volpe JJ. Trastornos neuromusculares: afección de músculos y trastornos restringidos. Neurología del recién nacido. $4^{\mathrm{a}} \mathrm{ed}$. México: Mc Graw Hill Interamericana; 2003: p.750-751.

17. García S, González H, Díaz B, Arenas A. Tortícolis Congénita: Incidencia y actuación fisioterápica en neonatos con contractura en el esternocleidomastoideo. Fisioterapia 2003; 25(1): 6-14.

18. Hong BY, Ko YJ, Kim JS, Ok EJ, Hwang Y, Kim HW. Sternocleidomastoid ultrasonography data for muscular torticollis in Infants. Muscle Nerve 2013; 48: 100-104. 